\section{Microabrasión dental para pacientes odontopediátricos: Una alternativa estética}

Dental microabrasion in children: An esthetic alternative

\section{Resumen}

La microabrasión dental es un procedimiento efectivo y conservador frente a los defectos superficiales del esmalte. Las manchas blancas del esmalte dental, aparecen frecuentemente en los niños por caries de esmalte inactiva, hipoplasias y fluorosis. Cuando estas manchas afectan a los dientes anteriores producen un efecto antiestético. Se describe dos casos clínicos de microabrasión del esmalte utilizando H3PO4 al 37 \% y piedra pómez, mediante una técnica mecánica (micromotor), en un niño de 7 ańos con diagnóstico de hipoplasia del esmalte y otro niño de 7 años con diagnostico de caries de esmalte inactivo, en ambos casos la manchas blancas fueron localizados en el sector anterosuperior. El objetivo fue comparar la efectividad de la microabrasión sobre manchas superficiales del esmalte utilizando el ácido ortofosfórico al 37 \% mediante el empleo de diferentes aditamentos en la técnica mecánica. Se observó que después de realizar una primera sesión de microabrasión, el resultado más eficiente se obtuvo con el uso de copas para pulir resina y piedra de acrílico elaborada respecto al grupo que usó copas de caucho para profilaxis, pero estas diferencias estéticas fueron desapareciendo en los controles posteriores.

\begin{abstract}
The dental microabrasion treatment is an effective and conservative procedure against enamel surface defects. The white spots appear frequently in children because inactive enamel caries, hypoplasia and fluorosis. When these spots affect anterior teeth produce an aesthetic effect. We describe two cases of enamel microabrasion of the upper incisors for both cases using $37 \% \mathrm{H}_{3} \mathrm{PO}_{4}$ and a mechanical technique (micromotor) in a 7 year old child with enamel hypoplasia and a 7 year old boy with inactive enamel caries. The aim of study was to compare affectivity of enamel microabrasion with $37 \% \mathrm{H}_{3} \mathrm{PO}_{4}$ and pumice using different gadgets for this mechanical technique. After the first microabrasion session, the best result was the one using resin polishing burs and home made acrylic pads respect to the group which use rubber points, but these differences did not longer appeared in the afterward sessions.
\end{abstract}

\section{Introducción}

La microabrasión es un método novedoso y conservador de la estructura dentaria frente a defectos superficiales del esmalte (caries dental, fluorosis e hipoplasia del esmalte), ${ }^{1,2}$ el cual consiste en eliminar las manchas del esmalte mediante una ligera abrasión de este y respetar el esmalte sano situado por debajo.

El primero en describir esta técnica fue el doctor Walter Kane en 1916, él utilizó el ácido clorhídrico al $36 \%$ y calor para eliminar las manchas cafés de los dientes. Posteriormente en 1984, McCloskey modificó esta técnica y usó el ácido clorhídrico $(\mathrm{HCl})$ al $18 \%$ sin el uso del calor. ${ }^{1,3}$ Luego Croll y Cavanaugh en 1986 desarrollaron una técnica de aclareamiento por medio de la microabrasión, con una aplicación de una mixtura de ácido clorhídrico al $18 \%$ y piedra pómez extrafina, en igual concentración utilizando un palillo de madera (técnica manual). ${ }^{4}$ Los resultados fueron buenos y duraderos, pero la técnica presentaba el inconveniente de trabajar con un ácido fuerte sin unión con la piedra pómez ofreciendo riesgos de al- canzar la mucosa causando quemaduras en el paciente, además de necesitar un tiempo largo de trabajo. 5

En el año 1995, Mondelli y col, señalaron que el uso de microabrasión con $\mathrm{HCl}$ al $18 \%$ proporciona resultados estéticos excelentes utilizando un número reducido de sesiones clínicas, sin embargo esta sustancia es un ácido fuerte y agresivo que exige cuidados especiales para evitar quemaduras químicas en la mucosa del paciente y en los dedos del operador, pero el emplear el ácido ortofosfórico al $37 \%$ asociado a piedra pómez en la proporción de 1:1, parece ser una alternativa segura y eficiente para este tipo de tratamiento, presenta la ventaja de ser una sustancia disponible en los consultorios odontológicos, además esta mixtura es fácilmente aplicable en relación con los otros materiales por su consistencia más densa, disminuyendo así el escurrimiento de la mezcla cuando se utiliza la copa de caucho. $^{6}$

Existen múltiples factores que se debe tomar en cuenta al momento de considerar el empleo de esta técnica, todos se basan en el conocimiento de los procedimientos y de los diferentes tipos de defectos y colo-
Caso Clínico

\section{María Álvarez Páucar ${ }^{1}$ Katherine Quiroz Gonzales² Vania Rodríguez Cruces ${ }^{2}$ Ross Mary Castelo Obregón²}

Docente Auxiliar del Departamento Académico de Estomatología Pediátrica de la Facultad de Odontología - UNMSM.

Alumnas del 4to año de la Facultad de Odontología de la UNMSM.

\section{Correspondencia:}

María Angélica Alvarez Páucar

Dirección: Facultad de Odontología de UNMSM

Germán Amézaga s/n, Lima 1. Perú.

E-mail: angelicaalvarez1@hotmail.com

Palabras clave: Microabrasión del esmalte, manchas blancas, ácido ortofosfórico.

Keywords: Enamel microabrasion, white spots, ortophosphoric acid

raciones que pueden afectar a los dientes y sus estructuras. De esta forma se puede evaluar la necesidad primaria de ésta u otra técnica para tratar estos defectos dentro del concepto actual de la operatoria y estética dental, para conservar el máximo tejido sano y la necesidad de lograr efectividad en el tratamiento.

Antes de realizar el tratamiento de microabrasión del esmalte dental, se deben tomar en cuenta los límites de los defectos de la descalcificación cuya profundidad debe ser entre 0,1 y $0,2 \mathrm{~mm}$, en caso de profundizar más allá del esmalte, se debe restaurar el diente aplicando un compuesto fotopolimerizado de resina. La profundidad de la mancha podría diagnosticarse dependiendo del origen de la misma.

La microabrasión del esmalte mejora el aspecto externo por microrreducción de la superficie del esmalte. ${ }^{8}$ El desgaste que se realiza sobre el esmalte con esta técnica, aumenta con variables como: presión ejercida, tiempo y número de aplicaciones. ${ }^{7}$ Diversos estudios no hallaron efectos postoperatorios relevantes o casi nulos, 
después de la aplicación del compuesto como: ausencia de sensibilidad térmica postoperatoria en los dientes tratados. ${ }^{\text {? }}$ Además, el ácido utilizado no es capaz de penetrar la dentina, por lo cual no existe contacto alguno entre la dentina, tejido pulpar y tejido periodontal. ${ }^{1,10}$

La expectativa y la ansiedad desarrollada por los padres y del niño en relación con la estética facial del paciente odontopediátrico, puede comprometer el desenvolvimiento psicológico del niño perjudicando su autoestima. ${ }^{11}$ La técnica de microabrasión puede ser utilizada en niños mayores de siete años, siempre y cuando exista la estricta supervisión del profesional, asimismo, los padres o representantes del niño se encuentren involucrados en el tratamiento y exista un adecuado manejo conductual del paciente. ${ }^{1}$

Esta técnica no está indicada para la remoción de manchas profundas: manchas características de tetraciclinas, dentinogénesis imperfecta, desvitalización o terapia endodóntica, ${ }^{12}$ aunque la verdadera limitante de la técnica es la profundidad de la pigmentación y el grosor del esmalte (especialmente en los incisivos inferiores). ${ }^{12,13}$

A continuación se presentan dos casos clínicos, donde se aplicó ácido ortofosfórico al $37 \%$ y piedra pómez, para la microabrasión del esmalte. En el primer caso se comparó la acción que produ-

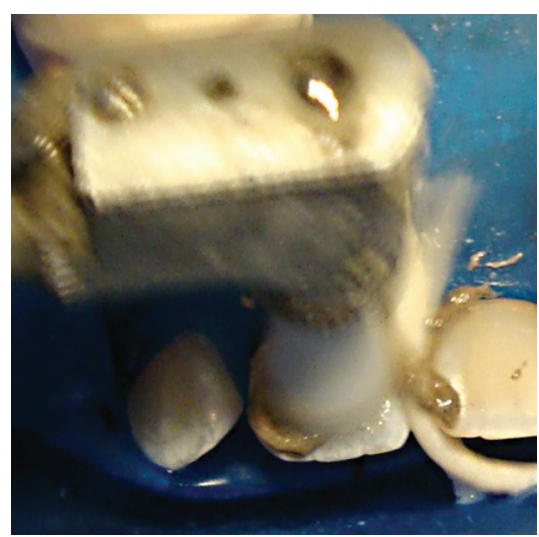

Fig. 1. Uso de copa de caucho.

cía al esmalte, el uso de copa de caucho para pulir resina con el grupo que usó copa de caucho para profilaxis (grupo control) y en el segundo caso se comparó la acción que producía el uso de la piedra de acrílico elaborada con el uso de la copa de caucho para profilaxis (grupo control). Luego se procedió a evaluar las superficies dentales tratadas, para hallar si hubo claras diferencias.

\section{Caso Clínico $\mathbf{N}^{0} 1$}

Paciente masculino de 7 ańos de edad acude a la clínica de la Facultad de Odontología de la Universidad Nacional Mayor de San Marcos, Departamento de Estomatología Pediátrica, donde se le efectúa el examen correspondiente y la elaboración de una minuciosa historia clínica con el empleo de los exámenes auxiliares correspondientes.

Diagnóstico: Mancha blanca debido a hipoplasia del esmalte localizada en la cara vestibular de las piezas: 11 y 21 .

Tratamiento: Microabrasión del esmalte dental utilizando una mezcla de ácido ortofosfórico al 37\% y piedra pómez.

Procedimiento: Después de informar a la madre el diagnóstico y del plan de tratamiento, se obtiene el consentimiento informado. Teniendo en cuenta las medidas de bioseguridad, se procedió a

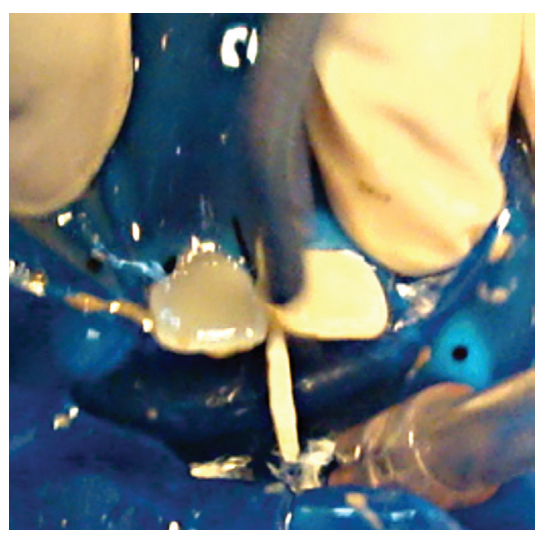

Fig. 2. Uso de copa para pulido. realizar el aislamiento absoluto sellando los márgenes cervicales de las piezas a tratar con barniz de copal para evitar el contacto de las mucosas con los químicos a utilizar. Luego de realizar la profilaxis dental, se procede a la preparación de la pasta, la cual se obtiene mezclando el ácido ortofosfórico al $37 \%$ más piedra pómez de grano ultrafino en la proporción 1:1, con el empleo de la técnica manual-mecánica: uso del micromotor.

Se aplicó la pasta sobre la superficie del esmalte, usando para la pieza 11, copa de caucho para profilaxis (Fig. 1) y para la pieza 21, copa para pulir resina (Fig .2), durante 10 segundos, seguido de un enjuague profuso con agua por 20 segundos (Fig. 3) Se realizaron 10 aplicaciones para cada pieza dental con ambas técnicas. Luego de secar ambas piezas dentales se realizó la remineralización con FNa al $2 \%$ neutro (Fig. 4) durante 4 minutos para cada diente.

\section{Caso Clínico $\mathrm{N}^{\circ} 2$}

Paciente masculino de 7 ańos de edad acude a la Clínica de la Facultad de Odontología de la Universidad Nacional Mayor de San Marcos, Departamento de Estomatología Pediátrica, donde se le realizó la elaboración de una correcta historia clínica con los exámenes correspondientes.

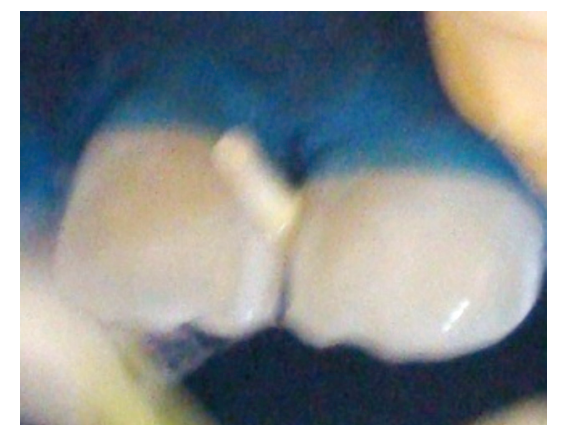

Fig. 4. Uso de FNa $2 \%$.

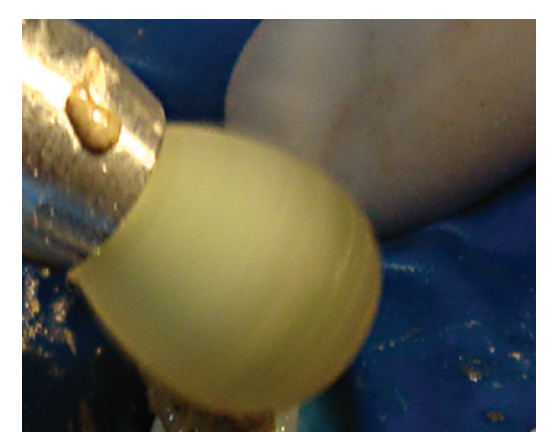

Fig. 5. Aplicación de la pasta con piedra de acrílico elaborada. 
Diagnóstico: Manchas blancas por caries de esmalte inactiva, esta lesión inicial está localizada en la cara vestibular de las piezas: 11, 21.

Tratamiento: Microabrasión del esmalte utilizando una mezcla de ácido ortofosfórico al $37 \%$ y piedra pómez extrafina.

Procedimiento: Igual que el caso anterior se obtuvo el consentimiento informado. Se realizó el mismo procedimiento, usando copa de caucho para profilaxis sobre la superficie dentaria 11 y para la superficie dentaria 21, se usó la piedra de acrílico elaborada, para lo cual se adicionó acrílico transparente sobre una copa de caucho, mediante la técnica de sal y pimienta (Fig. 5). Se aplicó la mezcla durante 10 segundos, seguido de un enjuague profuso de 20 segundos y se realizaron 10 aplicaciones para cada pieza dental, igual que el caso clínico anterior. Luego de secar ambas piezas dentales se realizó la remineralización con $\mathrm{FNa}$ al $2 \%$ neutro por 4 minutos para cada diente.

\section{Resultados}

En el Caso Clínico 1, primero se registró una foto inicial (Fig. 6), después se realizó la primera sesión donde se observó que ambas superficies tratadas mostraron diferencias en el color y aspecto. En la pieza 21, donde se aplicó la copa de caucho para pulir resina mostró un color más uniforme respecto a la pieza adyacente 11 donde se realizó la microabrasión con copa de caucho para profilaxis (Fig. 7).

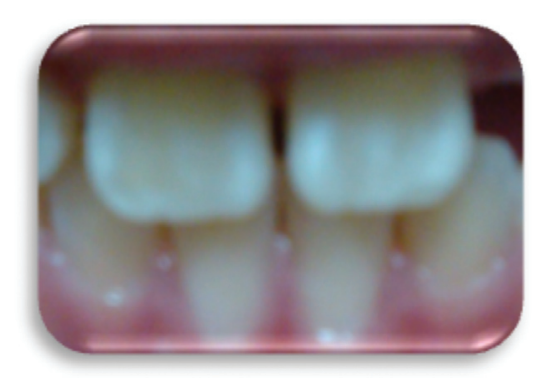

Fig. 6. Foto inicial.

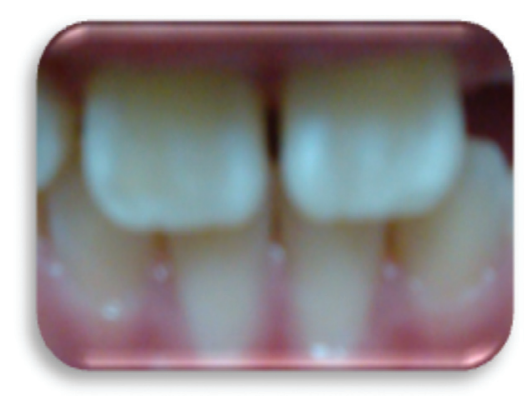

Fig 9. Foto inicial.
El paciente regresó a los 7 días, para el control respectivo y luego de examinar las superficies tratadas, se procedió a realizar una segunda sesión de microabrasión en ambas superficies dentales. Al final de la segunda sesión, se notó en ambas superficies tratadas, un aspecto y color uniforme, de tal manera que no hubo diferencia significativa, al efectuar el examen clínico (Fig. 8).

En el Caso Clínico 2, se registró primero una foto inicial (Fig. 9), después de realizarse la primera sesión se observó una clara diferencia favorable en el color y aspecto, cuando se aplicó el ácido con piedra de acrílico elaborada sobre la pieza dental 21, la cual mostró un aspecto más uniforme al compararlo con la pieza dental 11 donde se aplicó la mezcla con la copa de caucho para profilaxis, registrándose todavía irregularidad en la tonalidad del color (Fig. 10). Sin embargo, a los siete días cuando el paciente regresó para su control, luego del examen, esa mínima diferencia de color y aspecto que se había observado en la primera sesión, desapareció (Fig. 11). Por lo tanto, no fue necesario aplicar otra sesión.

\section{Discusión}

La microabrasión es un proceso efectivo para el tratamiento de las desmineralizaciones superficiales blancas y marrones incluyendo decoloraciones debido a fluorosis. ${ }^{13}$ Este procedimiento se realiza de manera rápida, efectiva y conservadora. Las causas más comunes de mancha blanca son caries dental

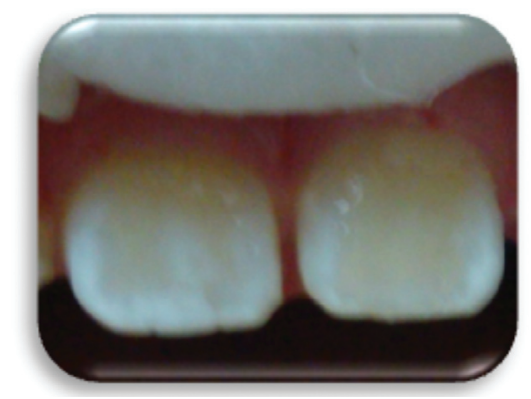

Fig. 7. Foto de primera sesión.

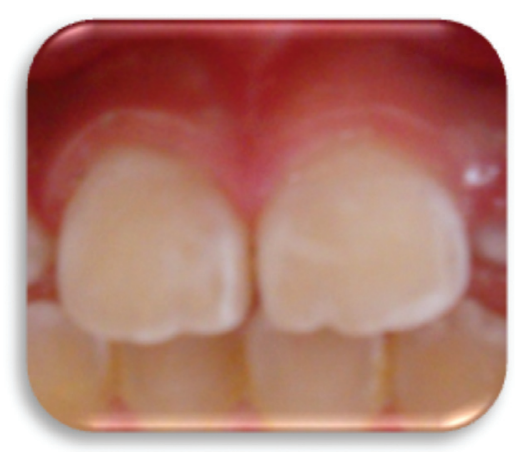

Fig 10. Foto de 1ra sesión. incipiente (caries de esmalte), fluorosis e hipoplasia del esmalte y tras los avances en la odontología surge una técnica no invasiva para la eliminación de estas manchas: la microabrasión.

Se plantearon muchas interrogantes respecto a la técnica de microabrasión, siendo la más común: ¿Qué técnica de microabrasión es más efectiva: la técnica con ácido clorhídrico $(\mathrm{HCl})$ o con ácido ortofosfórico $\left(\mathrm{H}_{3} \mathrm{PO}_{4}\right)$ ?

Para el presente trabajo se realizó la técnica de microabrasión del esmalte con ácido ortofosfórico, el cual es relativamente activo y actúa sobre la hidroxiapatita, extrayendo calcio que pasa a formar parte de la solución; como el esmalte sufre un ataque ácido se debe proceder a la aplicación tópica de fluoruros, el cual debe ser realizada usando flúor neutro para evitar posteriores alteraciones en la superficie dental. ${ }^{14} \mathrm{La}$ concentración más adecuada del ácido en el agua para lograr una correcta acción en el esmalte, es utilizando soluciones acuosas de ácido fosfórico entre el $32 \%$ y el $40 \%{ }^{3}$

La mezcla de $\mathrm{H}_{3} \mathrm{PO}_{4}$ y piedra pómez en una proporción de $1: 1$, produce acción ácido-abrasiva, ya que provee al diente un aspecto lustroso y brillante permanente, reduciendo las probabilidades de formación de caries en la superficie del mismo. ${ }^{9,10}$

Algunos estudios refieren que la acción de ambos ácidos presentan resultados exitosos similares y que el color del esmalte mejora con el tiempo, ${ }^{15}$ además

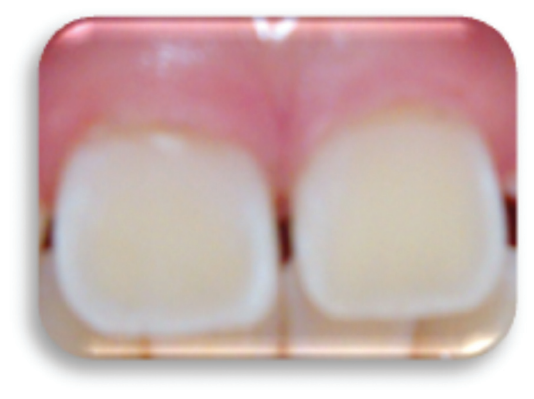

Fig. 8. Foto de segunda sesión.

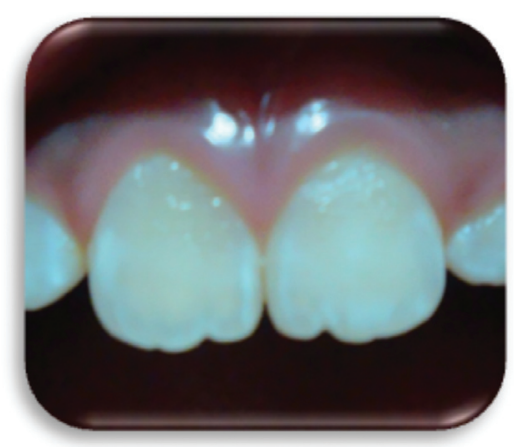

Fig 11. Foto de control.

Odontol. Sanmarquina 2009; 12(2):86-89 
los dientes mantienen la apariencia sana, de superficie brillante, sin sensibilidad post operatorias y las mejoras estéticas del tratamiento en el tiempo. ${ }^{16}$ Asimismo, cuando se compara rugosidad y pérdida de tejido, el empleo de ambos ácidos, eliminaron eficazmente la capa superficial del esmalte. ${ }^{17}$ Otro estudio refiere que el empleo del ácido ortofosfórico es una técnica de fácil uso y actúa selectivamente sobre los prismas del esmalte produciendo variaciones en los patrones de las superficies acondicionadas, sin embargo, el mismo estudio reveló que también produce un aumento de la rugosidad y deja una superficie áspera, pero que la pérdida del esmalte fue significativamente mayor cuando se utilizó el $\mathrm{HCl}^{18}$

En este contexto, el presente trabajo coincide con los estudios donde refieren que el empleo del $\mathrm{H}_{3} \mathrm{PO}_{4}$, fue menos agresivo tanto para la estructura adamantina, como en el caso de contacto accidental con la mucosa, piel y ojos del operador y/o del paciente como lo refirió Mondelli en $1995,{ }^{1}$ además de su alta disponibilidad en el consultorio odontológico, debido al uso frecuente en los procesos restaurativos adhesivos y ortodónticos y su menor costo. ${ }^{1,18}$

Por lo tanto, la microabrasión del esmalte, que emplea la técnica mecánica (baja velocidad: copa de caucho para profilaxis) es más rápida y aplica una fuerza constante; ${ }^{1}$ además es un tratamiento conservador para las descalcificaciones iniciales del esmalte, ya sea por fluorosis, hipoplasia dental y además, es una alternativa interesante para la remoción de manchas blancas superficiales, en casos de caries inactivas que muestran alteraciones en la micromorfología del esmalte; el efecto que produce este procedimiento es minimizado por la acción de los fluoruros y por el pulimento (eliminación de las estrías debido a la acción de la sustancia abrasiva). ${ }^{11} \mathrm{La}$ superficie lisa constituye un factor importante para la microabrasión del esmalte, al parecer se vuelve un área menos susceptible a la colonización de cierto tipo de bacterias como el $S$. mutans, ${ }^{1,5}$ asimismo, confirma la facilidad de colonización sobre las superficies dentarias rugosas y retentivas. ${ }^{1}$

La microabrasión del esmalte, otorga por sí sólo una superficie lisa y brillante, que se ve incrementada con el tiempo, a la vez que le confiere mayor capacidad de resistir la desmineralización y colonización de bacterias. ${ }^{5}$ Ya Silva y col. (1999), trataron las manchas oscuras con el empleo del ácido ortofosfórico y piedra pómez, más una punta diamantada de granulación fina (alta rotación), luego se pulió con piedra de óxido de aluminio y discos soflex y enseguida se aplicó flúor al $2 \% .{ }^{1}$ Pero, si la mancha era profunda, sólo en algunos casos se procede a la macro o megaabrasión, con remoción mecánica y su posterior restauración con resina neutra y traslúcida. ${ }^{12}$

\section{Conclusiones}

Se observó que después de realizar una primera sesión de microabrasión dental con el ácido ortofosfórico $\left(\mathrm{H}_{3} \mathrm{PO}_{4}\right)$, el resultado más eficiente y rápido, se obtuvo con el uso de copas para pulir resina y piedra de acrílico elaborada, respecto al grupo que usó copa de caucho para profilaxis, pero estas diferencias estéticas, tanto en la textura como el color fueron desapareciendo en los controles posteriores.

El diagnóstico constituye el elemento básico para la ejecución del tratamiento adecuado, nos referimos, cuando las manchas blancas son superficiales, especialmente en los casos de caries de esmalte inactivas.

\section{Referencias bibliográficas}

1. Silva SM, Lanza CR, De Oliveira FS, Machado MA. Tratamiento de hipoplasia del esmalte con la técnica de microabrasión en odontopediatría. Rev Odontológica Dominicana 1999; 5(1): 9-14.

2. Peruchi CM y col. O uso da microabrasão do esmalte para remoção de manchas brancas sugestivas de fluorose dentaria. Revista Odontológica de Araçatuba, 2004; 25(2): 72-77.

3. Sánchez M. Microabrasión del esmalte dental. Una alternativa para el tratamiento de la fluorosis. Reporte de un caso. Ustasalud odontología 2005; 4; 116-121

4. Croll TP, Cavanaugh R. Enamel color modification by controlled hydrochloric acid and pumice abrasion. Quintessence Int. 1986; 7 (2): 26-28.

5. Becerra VE, Gómez EA, Soler SL, Sampaio JM. Microabrasión del esmalte para el tratamiento de remoción de defectos superficiales, DENTUM 2005; 5(1):12-15.

6. Mendes RF, Mondelli J, De Freitas CA. Avaliação da quantidade de desgaste do esmalte dentário submetido à microabrasāo. Rev. FOB 1999; 7(1/2):35-40.

7. Dalzell D, Howes R, Hubler P: Microabrasión: effect of time, number of applications, and pressure on enamel loss. Pediatric Dentistry 1995 17(3):207-211

8. Croll TP. Enamel microabrasion followed by dental bleaching: case reports. Quintessence Int. 1992; 23: 317-321.

9. Croll TP. Enamel microabrasion: The Technique. Quintessence Int.1989; 20: 395-400.

10. Flaitz, C, Hicks MJ. Role of the acid etch technique remineralization of caries-like lesions of enamel: A polarized light and scanning electron microscopic study. J Dent. Child 1994; 3: 21-28.

11. Ramos CJ, Myaki I, Shintome LK, Arana-Chavez VE. Efeitos da Microabrasão sobre mancha branca de cárie inativa em dentes decíduos. Pesq Bras Odontoped Clin Integr, Joáo Pessoa 2006; 6(2): 149-154.

12. Natera GA, Uzcátegui GG, Peraza UI. Microabrasión del esmalte técnica para la remoción de manchas dentales. Acta Odontol. Venez 2005; 43(3). Disponible en: http:/ / www. actaodontologica.com. 02.04.2009.

13. Wong FS, Winter GB. Effectiveness of microabrasion technique for improvement of dental aesthetics. $\mathrm{Br}$ Dent J. 2002; 193(3):155-8.

14. Guedes-Pinto AC, Chedid S. Uso de los fluoruros en Odontopediatría. En: Guedes-Pinto AC y col. Rehabilitación Bucal en Odontopediatría. 1 ra ed. Colombia: Amolca: 2003. p.44-62.

15. Bezerra AC y col. Enamel opacities removal using two different acids: an in vivo comparison. J Clin Pediatr Dent. 2005;29(2):147-150.

16. Moncada CG, Urzúa a AI. Microabrasión del esmalte de incisivos superiores. Reporte Clínico. Revista Dental de Chile 2005; 96 (2): 25-27.

17.Schimidlin PR, Gohring TN, Schug J, Lutz F. Histological, morphological, perfilometric and optical changes of human tooth enamel after microabrasion. Am J Dent, San Antonio, 2003; 16 Spec. Issue, p. 4A-8A.16.

18. Meireles SS, Andre DA, Leida FL, bocangel JS, Demarco FF. Surface roughness and enamel loss with two microabrasion techniques. Journal Contemporary Dental Practice 2009; 10(1): 58-65.

Fecha de recepción: 18 diciembre 2009

Fecha de aprobación: 05 enero 2010 